\title{
Technologies de l'information et de la communication et pratiques informationnelles des médecins généralistes: quelles données en France?
}

\author{
Horence GONOD-BOSSIN
}

\begin{abstract}
Résumé Contexte: La médecine, comme bon nombre de secteurs, est largement concernée par l'essor des technologies de linformation et de la communication (TIC) et, en particulier, de l'Internet. De nombreuses études sintéressent à la nature des usages de ces nouvelles technologies au sein de la communauté médicale. But : Décrire l'historique des études internationales; faire l'inventaire des travaux conduits en France concernant les pratiques documentaires des médecins généralistes; analyser le comportement des médecins généralistes en France en matière de recherche documentaire; apprécier en particulier le niveau d'intégration des nouvelles technologies dans ces pratiques. Méthodes: Recherche documentaire systématique et analyse des travaux disponibles; enquête originale utilisant une approche qualitative de type compréhensif auprès d'un groupe de médecins généralistes de la région lyonnaise. Résultats: Notre recherche bibliographique a révélé la pauvreté des données françaises. Les études se limitent souvent à des initiatives institutionnelles ou journalistiques difficiles à exploiter sur le plan scientifique. Quelques travaux universitaires complètent le peu d'informations disponibles. Il s'agit dans la plupart des cas d'études quantitatives dont les données ne sont pas toujours comparables ni généralisables. Les résultats de notre approche qualitative montrent que l'informatique est utilisée pour la gestion administrative du cabinet mais qu'elle n'a pas fondamentalement modifié les pratiques documentaires des médecins généralistes; certains facteurs influençant l'usage des TIC au cabinet médical sont identifiés.
\end{abstract}

Mot clés Technologies de l'information et de la communication; innovation technique; internet médical; pratiques informationnelles; pratiques médicales; formation médicale continue; formation à distance; médecin généraliste.

Abstract Context: Medicine, as most of others fields, is concerned by the development of new information technologies (NIT) and Internet. Many studies aimed to explore the use of computer and Internet in the physicians' community. Aim: To describe the history of the international studies about this topic; to make an inventory of the works carried out in France concerning the documentary practices of the general practitioners; to analyze the behavior of the general practitioners in France as regards information retrieval; to appreciate particularly the level of integration of new technologies in these practices. Methods: Systematic bibliographical databases query and analyzing of available studies; original investigation using a qualitative approach near a group of general practitioners of the Lyon's area. Results: Searching bibliographical databases revealed the poverty of the French data. Studies are often limited to institutional or journalistic initiatives which are quite difficult to explore in a scientific way. Some university students' works were also available. Most of the cases were quantitative studies of which the data are not always comparable nor generalizable. The results of our qualitative approach show that NIT are used for the administrative management of the cabinet but that they basically do not modify the documentary practices of the general practitioners; some factors influencing the use of the NIT to the medical cabinet were identified.

Key words New information technologies; technical innovation; medical Internet; information behaviour; medical practices; continuing medical education; e-learning; general practitioner.

Pédagogie Médicale 2005; 6: 169-177

Correspondance: Horence Gonod-Boissin - Ecole Nationale Supérieure des Sciences de l'Information et des Bibliothèques (ENSSIB) - Laboratoire URSIDOC / DOCSI - 17/21 Boulevard du 11 Novembre 1918 - 69623 Villeurbanne Cedex Tél: 0472444343 - Fax:04 72444344 - mailto: boissin@enssib.fr 


\section{Nouvelles Technologies Éducatives}

\section{Introduction}

L'engouement à utiliser le Web, la messagerie électronique, les forums ou les listes de discussion fait le succès d'Internet que l'on connaît aujourd'hui. La croissance exponentielle de l'Internet à partir de 1995-1996 a permis un véritable essor dans bon nombre de secteurs, y compris en médecine. Cet essor concerne essentiellement l'offre de services. Le «boum » des sites Internet dédiés aux patients, en particulier aux Etats-Unis, illustre l'engouement du public pour ce média. De nombreux travaux récents, notamment anglo-saxons, s'intéressent à ce phénomène et à ses répercussions sur le rapport à la santé des patients ${ }^{1,2}$. Dans ce travail, nous avons cherché à analyser le comportement des médecins généralistes en France en matière de recherche documentaire. Il s'agissait d'apprécier l'intégration des technologies de l'information et de la communication (TIC) dans les pratiques informationnelles. Au préalable, nous avons souhaité rassembler les données recueillies par d'autres chercheurs autour de ces questions, tant en France qu'à l'étranger, afin d'optimiser les options méthodologiques de notre propre investigation. Nous avons défini l'activité d'information comme étant l'acquisition et le renouvellement des connaissances utiles pour procurer des soins adaptés au patient. Le cas du médecin généraliste nous semblait particulièrement intéressant en raison de son isolement professionnel fréquent. Les nouveaux outils d'accès à l'information pouvaient donc le séduire en lui offrant la possibilité de rompre cet isolement. Il paraissait intéressant d'observer comment le médecin vit son isolement lorsqu'il doit faire l'acquisition de son parc matériel, lorsqu'il doit le maintenir en bon état de marche et lorsqu'il doit s'y former.

Larticle retrace dans un premier temps l'historique des études du comportement documentaire des médecins, des années 60 à nos jours. Dans un deuxième temps, il s'attarde sur quatre études réalisées en France depuis la fin des années 90. Enfin, nous présentons quelques résultats de notre propre investigation qui a été menée auprès d'un panel de médecins généralistes de la région lyonnaise en 2003.

\section{Historique des études d'usages}

\section{Méthode et stratégie de recherche documentaire}

Dans un premier temps, nous avons effectué une recherche bibliographique dans la base de données Medline à l'aide de l'interface PubMed, afin de repérer les travaux décrivant les pratiques documentaires des médecins généralistes dans le monde entier. Aucun des motsclés Mesh ne correspondait vraiment à notre problématique hormis le mot-clé "family physician". Nous avons finalement effectué notre recherche dans le titre avec l'équation suivante: (physician+ OR doctor+ OR practi* $O R$ medicine) $A N D$ (Internet $O R$ information $A N D$ (need+ OR seek* OR search* OR source+ OR use* OR using OR behavio* OR medical)). Les caractères " + " et "* " désignent respectivement la prise en compte des pluriels et les troncatures. Nous avons restreint la recherche à $M E D-L I N E$ et à la mention "Human ». Dans ces conditions, notre requête a sélectionné 549 notices bibliographiques. Afin de limiter les résultats aux données concernant le médecin généraliste, nous avons exclu les autres professions médicales et les patients (opérateur NOT) avec les termes: "nurs* OR dent* OR surg* OR specialist+ OR residen* OR hospital+ OR patient+ OR consumer+ ". Nous avons choisi de ne pas d'emblée restreindre la recherche en utilisant les termes de "family medicine " (médecin de famille) ou "general practitioner " (médecin généraliste), afin de ne pas perdre trop d'informations. Finalement, nous avons obtenu 400 notices. La même recherche dans OLDMEDLINE (données avant 1965) a rapporté 14 références. Finalement un tri manuel a permis de sélectionner les références les plus pertinentes à savoir près de 75 notices.

Les chiffres de 414 puis de 75 références bibliographiques révèlent la richesse des travaux conduits autour des pratiques documentaires des médecins. La moitié de ces études sont américaines. Près de la moitié restante concerne des données européennes, en particulier d'Europe du nord (Suède, Norvège, Allemagne). La recherche française n'apparaît pas dans notre sélection. Enfin les données concernant le reste du monde sont anecdotiques (moins de 10 notices sur 75) et proviennent de la Nouvelle Zélande, d'Australie, d'Inde et du Canada. Afin de vérifier la pauvreté des données françaises, nous avons réalisé une recherche rapide et plus large dans les bases de données EMBASE et LISA; la recherche française n'y est pas mieux représentée.

\section{Les différentes générations d'étude d'usages dans le monde}

Les études des pratiques informationnelles des médecins débutent véritablement vers la fin des années 80. Avant cette date, nous trouvons plutôt la trace des premières 


\section{Technologies de linformation et de la communication...}

revues bibliographiques concernant les études d'utilisation des supports d'information ${ }^{3,4}$. Alors que leur nombre augmente, les études d'usages introduisent nettement la notion de " comportement documentaire des médecins " 5 . Elle portent d'abord un regard extérieur sur les outils d'accès à l'information considérés comme des objets techniques. Puis, la vision devient plus interne et on prend en compte le médecin et son contexte ${ }^{6}$ : il devient l'" acteur social manipulant l'objet technique $"^{7}$. Les travaux des années 80 s'intéressent aux aspects comportementaux des médecins pour chercher des références de documents au titre d'une activité spécifique: les soins, l'enseignement ou la recherche. Certains résultats révèlent à cette époque l'importance de la communication informelle entre médecins pour la recherche d'un avis ou d'une conclusion médicale ${ }^{8}$. Dans les années 90, de nombreux travaux s'intéressent au besoin d'information des médecins de famille', en particulier dans les zones rurales ${ }^{10}$. Avec l'avènement des TIC, on introduit la notion de "sources d'information préférées des médecins $»^{11,12}$. Les travaux de Haugg $^{12}$ aux Etats-Unis ont largement influencé les bibliothèques médicales. Ces dernières se sont référées aux conclusions de ses études pour mettre en place de nouvelles politiques documentaires qui prennent en compte les besoins réels et les habitudes documentaires des médecins. Haug montre notamment que les médecins préferent utiliser, en premier lieu, une information médicale issue des revues sur support papier et en second lieu des livres. Il confirme l'importance de la concertation avec les confrères de la même spécialité. Par la suite, les progrès importants enregistrés dans le secteur de l'édition électronique constitueront un tournant décisif dans l'apparition de nouveaux usages chez les médecins. On met en évidence l'usage de l'Internet comme un outil participant à la définition et à la mise en place d'un dispositif d'information et de communication. Celui-ci viserait à aider le médecin dans ses activités quotidiennes: l'Internet médical est étudié en tant que "co-constructeur d'un système d'information et de communication en médecine $"^{7}$. Vers la fin des années 90, on s'attache de plus en plus à l'analyse de l'utilisation d'Internet dans la profession. Ce nouveau moyen de communication faciliterait la disponibilité de l'information médicale : de nombreux services d'information et des bases de données sont accessibles sur des sites Web spécialisés. Le nouveau média présente toutefois l'inconvénient du manque de contrôle des informations ${ }^{13}$. Certains auteurs insistent aussi sur les avantages procurés par l'Internet dans le secteur spécifique de la for- mation médicale continue (FMC) ${ }^{14}$, à savoir une autonomie accrue et la possibilité d'accéder à des modules de formation depuis son cabinet. Des études conduites à l'étranger ces cinq dernières années se sont intéressées à l'intégration des TIC - et en particulier de l'Internet Médical - dans les activités quotidiennes des médecins. La plupart sont d'origine anglo-saxone. Certaines sont menées à l'échelle d'une nation voire de plusieurs; c'est le cas des enquêtes conduites par le Boston Consulting Group en Europe et aux Etats-Unis ${ }^{15,16}$. La méthode utilisée est généralement de type quantitatif avec des échantillons de taille variable, souvent de plusieurs centaines de médecins. La majorité de ces travaux axent leur problématique sur le comportement des médecins et sur l'intégration de l'outil Internet dans leur activité d'information quotidienne ${ }^{17,18}$. L'activité la plus étudiée est souvent l'activité hospitalière et de recherche ${ }^{19,20}$. Les résultats de ces récentes études révèlent un usage croissant des TIC, notamment du courrier électronique, de l'Internet Médical et des bases de données. L'usage grandit notamment au sein des communautés de médecins chercheurs ou hospitaliers: leur activité de recherche les oblige à s'informer des travaux scientifiques et donc à consulter les sites d'accès aux revues électroniques notamment. D'un point de vue géographique, l'intégration des nouvelles technologies est plus marquée aux Etats-Unis. Des médecins ont recours à la prescription électronique et considèrent que ces pratiques améliorent la qualité des soins et satisfont les patients ${ }^{16}$. Cette conclusion est toutefois à nuancer: les usages de l'Internet médical sont encore très hétérogènes. Les médecins ne sont pas préparés à utiliser tous ces outils pour se former en continu ${ }^{21}$. La tendance est désormais de comprendre ce qui favorise ou freine l'acceptation du nouveau média.

\section{Les données concernant le contexte français}

\section{La pauvreté des données disponibles}

Comme nous l'avons déjà évoqué, la recherche française sur le comportement documentaire des médecins généralistes est rarement visible dans les bases de données internationales. Des statistiques officielles concernant ces usages ne sont pas non plus disponibles. Pour pallier ce problème, nous avons orienté nos recherches vers les bibliothèques universitaires médicales [Bibliothèque inter-universitaire de médecine (BIUM)], les syndicats de médecins, les institutions (Ministère de la santé, Observatoire régional de la 


\section{Nouvelles Technologies Éducatives}

santé), l'Union professionnelle des médecins libéraux (UPML) et des écoles spécialisées [Ecole nationale supérieure des sciences de l'information et des bibliothèques (ENSSIB), Ecole nationale de santé publique (ENSP)]. Nous avons exclu les enquêtes de type journalistique et les sondages, toujours difficiles à exploiter dans un cadre de recherche.

Nombreuses sont les études qui concernent le thème de l'informatisation dans le secteur médical (près de 400 résultats pour la seule recherche dans le catalogue de la BIUM avec le sujet « informatique »). Mais beaucoup plus rares sont celles qui ont trait spécifiquement au comportement documentaire des médecins généralistes : on a retrouvé une dizaine de travaux universitaires (thèses de doctorat en médecine) et deux grandes enquêtes d'ampleur nationale conduites par l'Institut de recherche et de documentation en économie de la santé (IRDES, ex-CREDES) et l'Observatoire européen des technologies de communication (OTEC). Il s'agit généralement d'enquêtes de type quantitatif avec des échantillons de taille très variable.

Nous avons choisi de retenir les enquêtes les plus récentes, c'est-à-dire datant au plus de la fin des années 90 , soit de 1998/1999 à nos jours. C'est en effet à cette époque que les médecins français ont été contraints de s'équiper d'un ordinateur pour télétransmettre les feuilles de soins électroniques (ordonnances Juppé de 1996). Mesurer l'intégration des TIC dans les pratiques d'information des médecins avant cette date nous paraissait donc peu pertinent. Sur le plan régional nous avons finalement sélectionné deux travaux universitaires réalisés en RhôneAlpes $^{22,23}$. Au niveau national nous avons retenu les deux enquêtes de l'IRDES et de l'OTEC ${ }^{24,25}$. Ces quatre enquêtes nous ont aidés à illustrer le caractère hétérogène des données disponibles en France.

\section{Des données difficiles à comparer...et à généraliser}

Les caractéristiques de chacune des enquêtes retenues sont décrites dans le tableau 1.

Le tableau 1 révèle l'hétérogénéité des données issues des quatre enquêtes. Cela concerne le public cible: il n'est pas toujours constitué uniquement de médecins généralistes. L'objet d'étude est également très différent d'une étude à l'autre. Par exemple, l'étude de l'IRDES porte plus précisément sur "l'apport de l'informatique dans la pratique médicale ». L'objectif de cette enquête était d'établir des recommandations pour l'amélioration des logiciels médicaux. L'utilisation de l'informatique pour la recherche d'information ne constitue qu'un petit volet de l'étude. De plus, la manière de solliciter les médecins est très différente d'une enquête à l'autre. Ainsi, l'enquête IRDES est basée sur l'expérience de médecins volontaires qui ont bénéficié de l'aide à l'informatisation dans le cadre du Fonds de réorientation et de modernisation de la médecine libérale (FORMMEL). L'enquête a été conduite via Internet et/ou via le Réseau Santé Social, elle n’a donné lieu à aucun échange sous forme papier. Pour les deux enquêtes régionales en revanche, un questionnaire a été envoyé aux médecins, volontaires ou non. Enfin, la méthodologie est généralement celle de l'enquête quantitative mais les échantillons sont de taille très variable. L'enquête de l'OTEC comprend un volet qualitatif dont les données n’ont pas pu être analysées. Le chercheur n’a en effet pas pu obtenir ces données détenues par l'OTEC. En conclusion, au vu de cette hétérogénéité des données, il semble difficile de les comparer systématiquement et encore plus de les généraliser. Toutefois, nous tenterons ciaprès d'énoncer quelques-uns des résultats obtenus dans les différentes enquêtes françaises.

\section{Quelques résultats}

En France, l'informatisation des cabinets médicaux semble être une tendance récente et régulière encouragée par les politiques de santé. L'ordonnance Juppé du 24 avril 1996 (Journal Officiel du 25/04/1996) concernant l'informatisation des cabinets médicaux, ainsi que la création du FORMMEL en 1998, ont largement favorisé l'équipement des médecins libéraux: en 1996, sur les quelques 60400 généralistes exerçant en France à titre libéral, environ 20000 sont équipés d'un micro-ordinateur ${ }^{26}$, soit un taux d'informatisation de 33-34\%. Au printemps 1999, $65 \%$ des médecins généralistes sont informatisés. En outre, certaines enquêtes montrent que l'utilisation des ressources informatiques au cabinet concerne principalement la gestion du dossier médical (99\% des médecins de l'enquête de l'IRDES). D'autres fonctions émergent ensuite, telles que le traitement de texte, la gestion comptable du cabinet, l'aide au diagnostic et à la prescription, la messagerie électronique, l'optimisation économique des actes et prescriptions et enfin l'accès à des bases de données via Internet.

Un autre point important concerne le besoin de fiabilité des médecins vis-à-vis de la recherche d'information. Malgré une augmentation des connexions à Internet et bien que la majorité des médecins ressentent le besoin de 


\begin{tabular}{|c|c|c|c|c|c|c|c|c|}
\hline \multirow{5}{*}{ 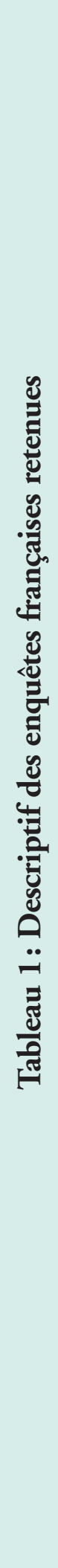 } & 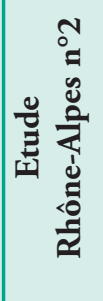 & 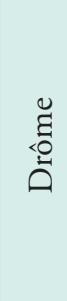 & 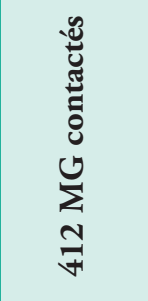 & 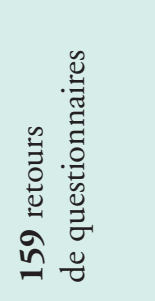 & $\neg$ & ఫ్రి & 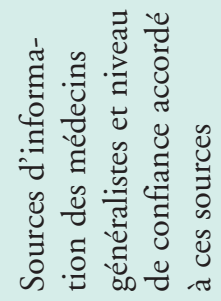 & \multirow{5}{*}{ 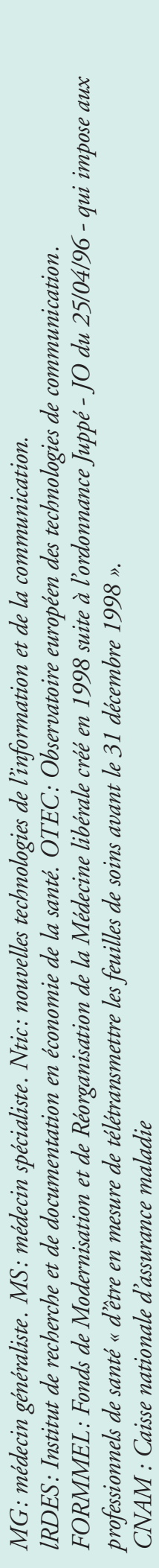 } \\
\hline & 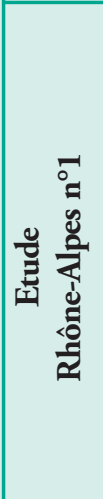 & 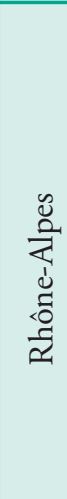 & 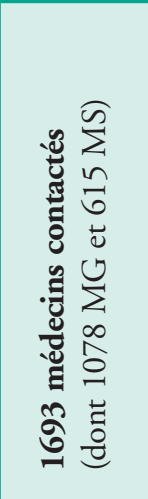 & 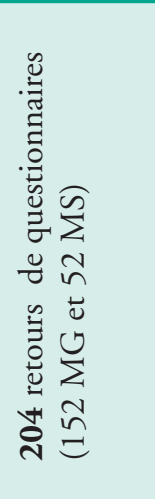 & $\neg$ & 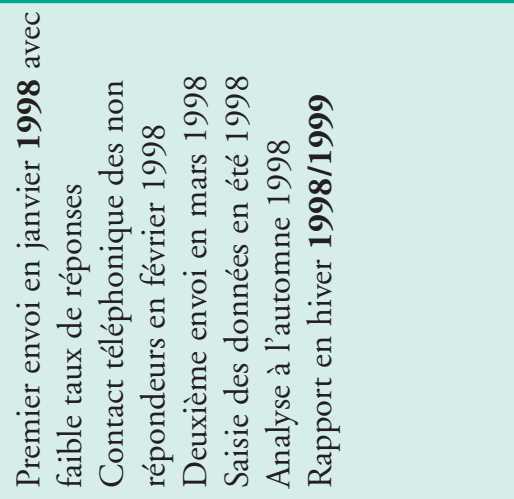 & 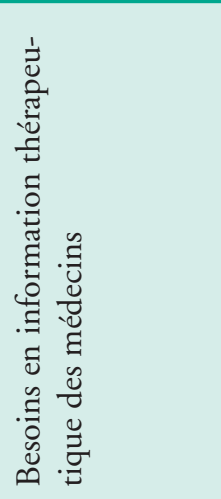 & \\
\hline & 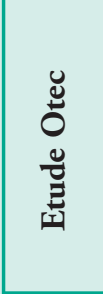 & 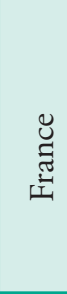 & & તิ̀ & - & 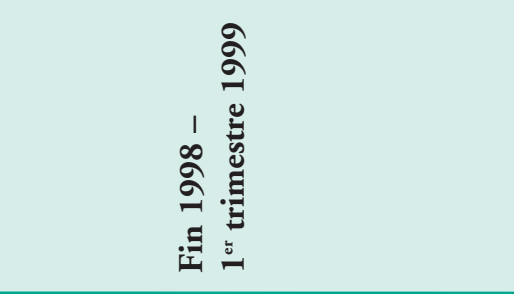 & 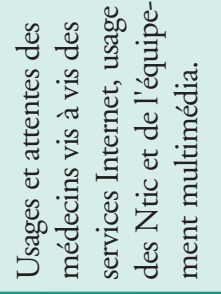 & \\
\hline & 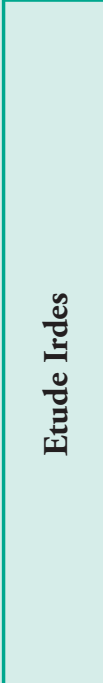 & 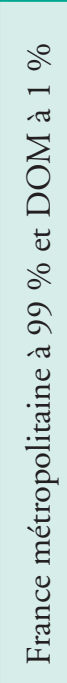 & 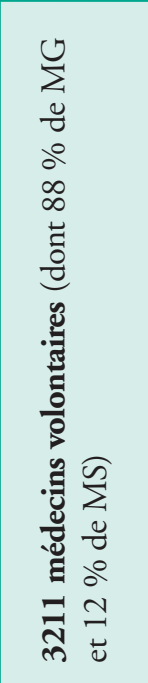 & 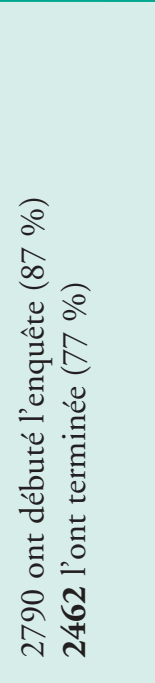 & 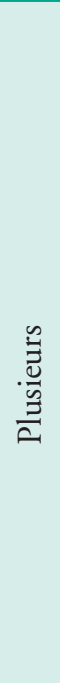 & 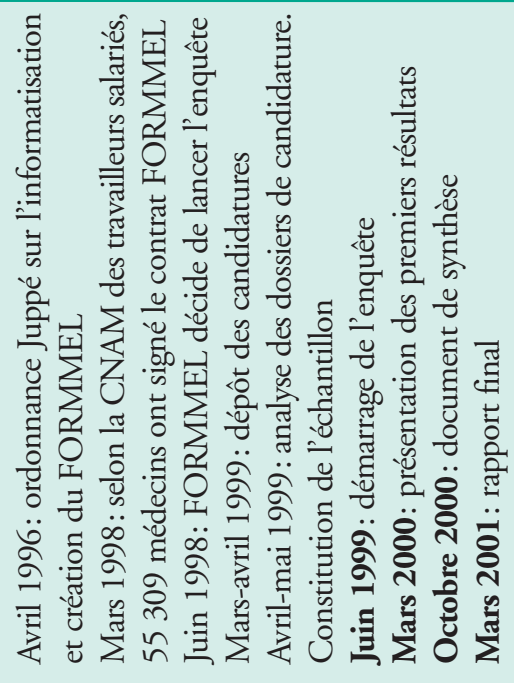 & 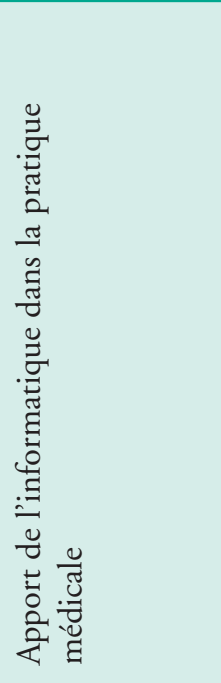 & \\
\hline & 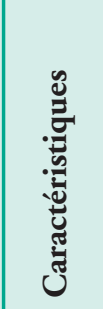 & 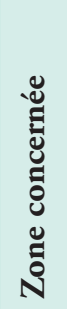 & 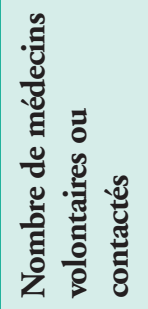 & 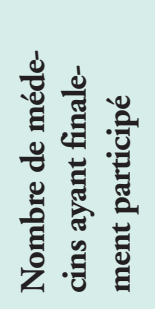 & 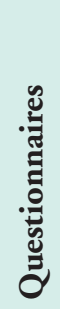 & 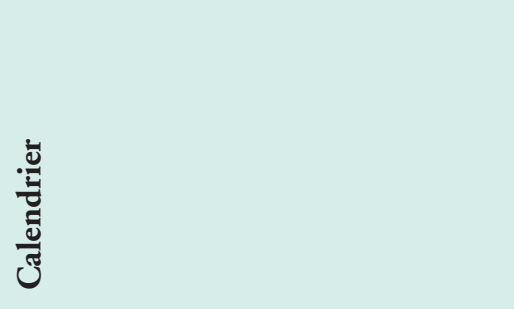 & 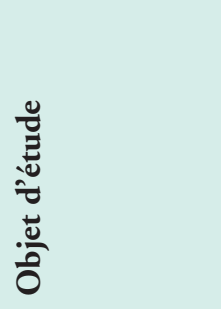 & \\
\hline
\end{tabular}




\section{Nouvelles Technologies Éducatives}

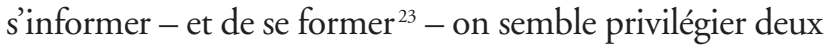
sources: sa bibliothèque personnelle d'une part, l'avis de ses confrères, d'autre part. La bibliothèque personnelle est composée de presse médicale de formation et d'ouvrages de référence; les confrères sont indifféremment spécialistes ou généralistes. La messagerie électronique, quant à elle, connaît un certain essor ${ }^{24}$. Laccès au savoir demeure, cependant, une des attentes majeures des médecins vis à vis des applications de la télémédecine (télé-formation et télé-informations ${ }^{27}$. On craint toutefois une perte de confidentialité et une mauvaise fiabilité des sources, primordiale dans le domaine médical. La transmission d'informations concernant les patients semble, de ce fait, freinée ${ }^{24}$. Autre conséquence, les médecins semblent accorder plus de confiance aux sites institutionnels et aux sites indiqués par la presse de formation et les collègues. En effet, l'IRDES confirme la consultation de plus en plus forte des bases de données médicales reconnues et accessibles via des sites institutionnels: bases du Centre hospitalier universitaire (CHU) de Rouen, MEDLINE, bases du site du CHU de Rennes, site de la Haute autorité de santé (HAS, ex ANAES), site du Conseil de l'Ordre, site Web du dictionnaire des spécialités médicales Vidal, etc. En général, quelle que soit la nature des bases interrogées, le médecin effectue une requête pour répondre à un cas précis ou également dans le cadre de la FMC ${ }^{24}$. Les bases de données bibliographiques sont plus fréquemment interrogées dans un but de recherche ou pour préparer des publications mais les médecins généralistes sont généralement peu concernés par ce type d'activité, contrairement aux praticiens de $\mathrm{CHU}$ par exemple ${ }^{7}$.

Certains travaux notent les avantages des TIC énumérés par les médecins, notamment le gain de temps ${ }^{25}$, à condition de bien maitriser l'outil informatique. La relation soignant-soigné, quant à elle, est jugée améliorée selon les médecins équipés d'un ordinateur. Les médecins de l'enquête IRDES estiment en effet que l'informatique améliore la qualité de leur travail. Elle permet une meilleure gestion des données concernant le patient. Certains médecins non équipés redoutent quant à eux une altération de la relation médecin-patient ${ }^{26}$. Il est à noter également que les réticences vis-à-vis de l'informatique sont nuancées en fonction de l'âge et du sexe des médecins. Les plus jeunes semblent plus enthousiastes ${ }^{25,26}$; les femmes paraissent plus réticentes que les hommes. Enfin, les médecins qui ont une lourde charge de travail sont plus attirés par l'informatique pour le gain de temps éventuellement procuré. Certains médecins généralistes estiment que l'informatisation peut procurer des avantages collectifs de diverses nature ${ }^{25,26}$ : la rationalisation des dépenses de santé, l'amélioration des prescriptions, l'amélioration du diagnostic, le développement de la FMC, le développement des filières de soins, le gain de temps (sous condition d'une bonne maîtrise de l'outil) et l'amélioration de la relation médecin-patient.

Ces quelques résultats révèlent des tendances mais leur généralisation, nous l'avons dit, doit être prudente. Dans tous les cas, l'approche méthodologique quantitative dont elles sont issues ne permet pas de déterminer l'ensemble des facteurs ni les motivations d'usages ${ }^{28}$.

\section{Notre enquête auprès des médecins généralistes lyonnais}

\section{Le parti pris qualitatif de notre étude}

Dans ce contexte, nous avons décidé d'adopter une approche complémentaire afin de nous concentrer sur les aspects sociologiques des usages. L'objectif était de mieux comprendre les enjeux et les freins de l'intégration des TIC dans les pratiques d'information des médecins généralistes. Plusieurs questions ont émergé: quels facteurs influencent l'usage de l'information électronique par les médecins généralistes? L’abondance de sites web dédiés à la santé engendre-t-elle de nouveaux usages? La recherche d'information électronique est-elle intégrée au processus général d'informatisation du monde de la santé? Les anciens outils de recherche d'information sont-ils abandonnés au profit des nouveaux? Quelles sont les limites entre la sphère privée et la sphère professionnelle? Les relations au sein de la communauté médicale ont-elles été modifiées par l'arrivée des TIC au cabinet?

Pour tenter de répondre à l'ensemble de ces questions, l'approche qualitative a été choisie. Elle devait nous permettre de mieux cerner les pratiques et les comportements ${ }^{28}$. Nous avons mené des entretiens individuels au cabinet des médecins généralistes pour mieux observer et décrire leur contexte de travail. Les données ont été collectées de janvier à mars 2003. Nous avons utilisé le fichier des médecins de l'UPML pour construire notre échantillon. Un courrier détaillant l'objet de nos recherches a été envoyé à 200 médecins généralistes. Nous avons ensuite contacté 62 d'entre eux par téléphone pour fixer un rendez-vous; 32 ont accepté de nous rencontrer. Les entretiens ont été enregistrés puis retranscrits intégralement. Nous avons ensuite procédé à l'analyse 


\section{Technologies de linformation et de la communication...}

thématique de ces entretiens afin de révéler les sentiments exprimés par les médecins généralistes.

Lapproche qualitative a longtemps été critiquée pour son manque de rigueur scientifique. La question de la validité et de l'objectivité des données issues d'enquêtes qualitatives a souvent été posée. Guba et Lincoln, notamment, ont identifié quatre types de facteurs pour juger de la qualité des données ${ }^{29}$ (" truth value, applicability, consistency and neutrality "). D'autres auteurs ont appliqué ces critères aux enquêtes conduites auprès des professions de santé, des infirmières en particulier ${ }^{30}$. La méthode que nous avons choisie se base sur les principes anthropologiques d'observation, déjà utilisés dans le cadre de l'activité scientifique $^{31}$. Notre démarche s'appuie aussi sur les principes de l'entretien compréhensif défini par Kaufmann comme une " méthode adaptée à un usage artisanal plutôt qu'industriel qui permet la construction de l'objet scientifique " ${ }^{28}$. A la question de la rigueur, Kaufmann répond que " les méthodes qualitatives ont pour fonction de comprendre, de détecter des comportements, des processus plus que de décrire systématiquement, de mesurer ou de comparer ". C'est pourquoi nous parlerons par la suite de "l'ensemble des informateurs " plutôt que "d'échantillon » qui sous-entend la notion de représentativité. La recherche de représentativité statistique n’a en effet plus de sens ici puisqu'on privilégie "la variété, ainsi que l'expérience et la spécificité d'un individu dans un processus collectif " ${ }^{32}$. Toutefois, l'idéal aurait été de croiser les deux approches, quantitative et qualitative ${ }^{33}$. Dans notre cas il aurait fallu disposer de statistiques officielles d'usages; or nous l'avons vu, les données de ce type n'étaient pas disponibles.

\section{Principaux résultats}

Nous nous contenterons d'énoncer les résultats les plus importants de notre investigation. L'ensemble de notre analyse sera développé plus longuement dans un document dédié (thèse en cours).

Notre panel était constitué de 26 hommes et de 6 femmes. Ces dernières ont été plus difficiles à solliciter. Elles évoquaient un emploi du temps très chargé, devant « concilier activités professionnelle et familiale ». Lâge moyen des informateurs était de 48 ans, ce qui correspond approximativement à l'âge moyen des médecins généralistes en France. Pour environ la moitié d'entre eux, une activité de spécialité s'ajoute à l'activité principale de médecine générale (médecine du sport, homéopathie, animation de
FMC, etc.). Le niveau d'informatisation était élevé puisque 28 des médecins rencontrés étaient équipés d'un ordinateur. Cet équipement était plutôt récent (1999/2000) et a été vécu comme une contrainte: il était lié à la nécessité de télétransmettre les feuilles de soins électroniques (TFSE). Mais les médecins généralistes ont vu là une occasion de se connecter à Internet, " comme tout le monde ".

Dans les faits, l'informatique est utilisée pour la gestion administrative du cabinet (comptabilité, dossier médical, TFSE) mais n'a pas fondamentalement modifié les pratiques documentaires des médecins généralistes rencontrés. La maintenance du parc matériel est jugée lourde et coûteuse. La recherche d'information est majoritairement motivée par l'activité secondaire que nous avons évoquée précédemment. Elle fait encore appel aux circuits traditionnels d'accès à l'information, les médecins généralistes privilégiant leur bibliothèque personnelle et le réseau de confrères. Neuf des médecins rencontrés disent utiliser Internet pour rechercher une information médicale. Ils avouent aussi ne pas maittriser convenablement ces nouveaux outils. Ils connaissent mal les sites spécialisés en santé. En conséquence leurs recherches se sont souvent soldées par un échec. Les médecins généralistes de notre panel estiment donc qu'il est « difficile de trouver la bonne information sur Internet ». Par ailleurs, les relations au sein de la communauté médicale n'ont pas été fondamentalement modifiées avec l'arrivée des TIC. Les réseaux de confrères sont ceux établis à l'issue des études universitaires ou bien ceux générés par les formations de groupe dans le cadre de la FMC. Les médecins généralistes de notre panel n'ont pas de pratique de FMC en ligne. Ils préferent les rencontres entre confrères, plus conviviales et idéales pour rompre leur isolement. Un responsable d'une association de FMC de Lyon rappelle que les formations de groupe sont importantes pour lutter contre le syndrome de burn-out des médecins. Pour lui, même les meilleurs TIC ne pourront procurer de tels bénéfices.

\section{L'avenir des TIC chez le médecin généraliste}

Quel avenir peut-on, dès lors, entrevoir pour les TIC au cabinet médical? Tout d'abord, le métier de médecin, rappelons-le, est avant tout un métier de contact. Les médecins généralistes actuellement en place, âgés en moyenne de 49 à 50 ans, ont été habitués à la formation en présentiel, à l'échange de points de vue entre confrères. Pour cette génération de médecins, il sera difficile de chan- 


\section{Nouvelles Technologies Éducatives}

ger les réflexes de formation et les pratiques d'information. D'ailleurs, le problème n'est pas tant le renouvellement des connaissances mais plutôt la confrontation des pratiques professionnelles. L'intérêt des TIC pourrait se situer à ce niveau en permettant le développement d'outils dédiés à l'évaluation des pratiques professionnelles (EPP). D'ailleurs, les récentes orientations légales du ministre de la santé (Philippe Douste-Blazy) prévoient un couplage $\mathrm{EPP} / \mathrm{FMC}$, avec EPP obligatoire tous les cinq ans. Encore faudra-t-il que des changements s'opérent chez les médecins généralistes. Actuellement, la profession est boudée: seulement $29 \%$ des médecins en fin d'études choisissent la médecine de cabinet. Les médecins se raréfient et ceux qui demeurent ont des emplois du temps surchargés. Il sera donc difficile d'intégrer des modules de FMC classique et encore plus des modules de formation à l'usage des TIC!

\section{Conclusion}

Les travaux conduits auprès des médecins généralistes concernant leurs pratiques d'information sont donc nombreux. Mais les données spécifiquement françaises sont beaucoup plus rares et difficiles à exploiter dans un cadre de recherche. Notre propre investigation s'est attachée à déceler des comportements et des facteurs d'usages des TIC dans le cadre des pratiques d'information de médecins généralistes. Elle ne constitue nullement un aboutissement mais plutôt une proposition pour de nouvelles pistes de recherches autour de ces questions. Les TIC constituent en effet un des enjeux majeurs de la médecine de demain. Leur intégration dans les pratiques informationnelles des médecins généralistes pourrait révéler les mutations futures du métier de médecin de famille.

\section{Références}

1. Nabarette H. Linternet médical et la consommation dinformation par les patients. Réseaux 2002; 114 : 249-285.

2. Eysenbach G, Kohler C. How do consumers search for and appraise health information on the world wide web? Qualitative study using focus groups, usability tests, and in-depth interviews. BMJ 2002 ; 324: 573-577.

3. Boeck W. Annoted bibliography of studies on the flow of medical information practitionners. Bethesda (Ma): Institute for Advancement of Medical Communication, 1961.

4. Sherrington AM. An annotated bibliography of studies on the flow of medical information to practitioners. Methods Inf Med 1965; 4 : 45-57.

5. Paisley WJ. Information uses and needs. Ann Rev Inf Sci Technol $1968 ; 3: 1-38$.

6. Strasser TC. The information needs of practising physicians in Northeastern New York State. Bull Med Libr Assoc 1978; 66:200-209.

7. Tietse S. Apport de l'internet dans la capitalisation des connaissances et conception d'un système d'information médicale autour de la médiation entre connaissances et actions des praticiens dans un hôpital universitaire. Lyon: UCBL, 2003. Thèse d'université.

8. Weinberg. Informal advice and information seeking between physicians. J Med Educ 1981; 56 : 174-180.
9. Bryant SL. The information needs and information seeking behaviour of family doctor. Health Info Libr 2004; $21: 84-93$.

10. Dee C. Information needs of the rural physician: a descriptive study. Bull Med Libr Assoc 1993; 81: 259-264.

11. Connelly DP, Rich EC, Curley SP, Kelly JT. Knowledge resource preferences of family physicians. I Fam Pract $1990 ; 30: 353-359$.

12. Haug JD. Physicianspreferences for information sources: a meta-analytic study. Bull Med Libr Assoc 1997; $85: 223-232$.

13. Xavier P. Utilisation du réseau informatique internet en médecine générale en France. Bordeaux:Bordeaux II, 1998. Thèse pour le diplôme d'état de docteur en médecine.

14. Pultar P. Internet et formation médicale continue en France. Poitiers: 1998. Thèse pour le diplôme d'état de docteur en médecine.

15. Flanagan A, Guy P, Larsson S, Saussois C. European physicians and the Internet. Boston: The Boston consulting Group, 2003.

16. Von Knoop C, Lovich D, Silverstein M.B, Tutty M. Vital signs: E-health in the United-Sates. Boston: The Boston consulting Group, 2003. 
17. Forrest $M$, Robb $M$. The information needs of doctorsin-training: case-study from the cairns library, University of Oxford. Health Libraries Review 2000; 129-135.

18. Nylenna M, Aasland OG. Primary care physicians and their information-seeking behaviour. Scand J Prim Health Care 2000 ; $18: 9-13$.

19. Laerum H, Ellingsen G, Faxvaag A. Doctor's use of electronic medical records systems in hospitals: cross sectional survey. BMJ 2001; $323: 1344-1348$.

20. Murray J, Carey E, Walker S. The information needs and information seeking behaviour of medical research staff. Health Libraries Review 1999; 16 : 46-49.

21. Shaughnessy AF. Are we providing doctors with the training and tools for lifelong learning? BMJ 1999; 319: 1280 .

22. Lambert R. Enquête sur les besoins en information thérapeutique auprès de 152 médecins généralistes et de 52 médecins spécialistes libéraux de la région RhôneAlpes. Lyon: Université Claude Bernard Lyon 1, 1999. Thèse pour le diplôme d'état de docteur en médecine.

23. Pholsena R. source d'information des médecins généralistes et niveau de confiance accordé à ces sources. Enquêtes auprès de 412 médecins de la Drôme. Lyon: Université Claude Bernard Lyon 1, 2000. Thèse pour le diplôme d'état de docteur en médecine.

24. CREDES (Centre de Recherche, d'étude et de documentation en économie de la santé), CSSIS (Conseil Supérieur des Systèmes d'Information et de Santé), FORMMEL (Fonds de Réorientation et de Modernisation de la Médecine Libérale). L'apport de l'informatique dans la pratique médicale libérale. Paris: CREDES, CSSIS, 2001. [On-line]. Disponible sur: http://www.irdes.fr/En_ligne/Rapport/rap2001/rapport Formmel.pdf
25. Plisson P, Wagner Ballon J. Les médecins usagers d'Internet: approche générale et analyse d'une étude menée auprès du corps médical par l'OTEC auprès de 3296 médecins. Tours: Université de Tours, 2000. Thèse pour le diplôme d'état de docteur en médecine.

26. Levy D, Bui DHD. Linformatisation des cabinets médicaux: faits et opinions à travers les réponses de 1720 médecins généralistes. Paris: John Libbey Eurotext, 1997.

27. Fraisse $S$ et al. La télémédecine dans le Rhône. Lyon: Université Jean Moulin Lyon 3, 2001. Etude réalisée pour le compte d'ASTRHO, du grand Lyon et du Conseil Général du Rhône par IFROSS de la Faculté de Droit.

28. Kaufmann JC. L'entretien compréhensif. Paris: Nathan, 1996.

29. Lincoln Y, Guba E. Naturalistic inquiry. Bervely Hills: Sage Publications, 1985.

30. Sandelowski $M$. The problem of rigor in qualitative research. Advances in nursing science (ANS), 1986; $8(3): 27-37$.

31. Latour B, Woolgar S. La vie de laboratoire. La production des faits scientifiques. Paris: La Découverte, 1988.

32. Mahé A. La communication scientifique en (r)évolution: lintégration des revues électroniques dans les pratiques informationnelles de chercheurs en sciences de la nature comme révélateur des mutations du modèle traditionnel de la communication scientifique. Lyon: Université Claude Bernard Lyon I, 2002. Thèse d'université.

33. Ranjard S. Evaluer la demande et les besoins en informations: pour des enquêtes croisées. Documentaliste Sciences de l'Information, $2001 ; 38: 14-23$.

Manuscrit reçu le 23 novembre 2004; commentaires éditoriaux formulés aux auteurs le 10 mars 2005; accepté pour publication le 19 mai 2005. 